\title{
Effect of autophagy induced by dexamethasone on senescence in chondrocytes
}

\author{
ENXING XUE ${ }^{1,2}, \mathrm{YU}_{\mathrm{ZHANG}}{ }^{2}, \mathrm{BING}_{\mathrm{SONG}}{ }^{1}, \mathrm{JUN}_{\mathrm{XIAO}}{ }^{1}$ and $\mathrm{ZHANJUN} \mathrm{SHI}^{1}$ \\ ${ }^{1}$ Department of Orthopedic Surgery, Nanfang Hospital, Southern Medical University, Guangzhou, Guangdong 510515; \\ ${ }^{2}$ Department of Orthopedic Surgery, The Second Affiliated Hospital and Yuying Children's Hospital of Wenzhou Medical University, \\ Wenzhou, Zhejiang 325000, P.R. China
}

Received August 3, 2015; Accepted August 8, 2016

DOI: $10.3892 / \mathrm{mmr} .2016 .5662$

\begin{abstract}
The aim of the current study was to explore the effects of dexamethasone (DXM) on autophagy and senescence in chondrocytes. Collagen II and aggrecan were examined in normal chondrocytes isolated from Sprague-Dawley rats. Following stimulation with DXM, LysoTracker Red staining, monodansylcadaverine (MDC) staining, green fluorescent protein-red fluorescent protein-light chain 3 (LC3) and western blotting were used to detect autophagy levels in the chondrocytes. Mechanistic target of rapamycin (mTOR) pathway-associated molecules were investigated by western blotting. Cell senescence was analyzed by senescence-associated (SA)- $\beta$-galactosidase $(\beta$-gal) staining. A dose-dependent increase in the number of autophagic vacuoles was observed in the DXM-treated chondrocytes, as demonstrated by LysoTracker Red and MDC staining. A dose-dependent increase in autophagosome formation was observed in the DXM-treated chondrocytes. Expression of LC3-II and beclin-1 was increased by DXM, in particular in the cells treated with DXM for 4 days. However, P62 expression was reduced as a result of treatment. SA- $\beta$-gal staining indicated that DXM increased cell senescence. Notably, DXM-induced cell senescence was exacerbated by the autophagic inhibitor 3-MA. Autophagy induced by DXM protected chondrocytes from senescence, and it is suggested that the mTOR pathway may be involved in the activation of DXM-induced autophagy.
\end{abstract}

\section{Introduction}

Osteoarthritis (OA) is a common disease, and its incidence rate in people over 25 years old is $14 \%$, with $\sim 400,000$ patients diagnosed with OA per year in the Catalonia region of

Correspondence to: Professor Zhanjun Shi or Professor Jun Xiao, Department of Orthopedic Surgery, Nanfang Hospital, Southern Medical University, 1838 North Guangzhou Road, Guangzhou, Guangdong 510515, P.R. China

E-mail:nfgk@sohu.com

E-mail: orthopaedxj@163.com

Key words: dexamethasone, chondrocyte, autophagy, senescence
Spain (1). Clinically, local intra-articular injection of DXM has an anti-inflammatory function, relieving the symptoms of knee OA, such as pain and swelling. However, long-term and repeated injections of DXM can disrupt the metabolic balance in chondrocytes, leading to the catabolism in the cartilage with increase of matrix metalloproteinases (MMPs), and can accelerate cell apoptosis and death. This may result in a reduction in chondrocyte number and further deterioration of articular cartilage in OA. Therefore, a novel method to maintain the survival of chondrocytes under DXM stimulation is required.

Autophagy, a self-protective mechanism, has been suggested to maintain cellular homeostasis by removing protein aggregates and damaged organelles by the fusion of autophagosomes and lysosomes. Previous studies have demonstrated that autophagy is a protective process preventing against chondrocyte apoptosis and cartilage degeneration $(2,3)$. In a mouse model, local injection of rapamycin, an inducer of autophagy, has been demonstrated to impede the progress of arthritis $(2,4)$. It is widely accepted that autophagy in mammalian cells can be adaptively activated by different stresses (5-7), therefore, it was hypothesized that DXM may be able to activate autophagy in chondrocytes.

Previous studies have demonstrated that the ability of joint tissues to restore the articular surface is reduced with age; patients with intra-articular fractures of the knee $>50$ years have a greater risk of developing OA than younger patients $(8,9)$. Age also increases the risk of post-traumatic OA in patients with anterior cruciate ligament tears (9). With the aging of cartilage, senescent chondrocytes may accumulate in the cartilage, leading to the loss of ability to maintain and restore articular cartilage effectively (10). A previous study additionally observed a clear correlation between increasing age and incidence of OA (11). Aging is the largest risk factor for $\mathrm{OA}$, and cell senescence serves an important role in the occurrence and development of OA. However, the exact mechanism involved in the effect of DXM on chondrocyte senescence remains unclear.

A previous study demonstrated that autophagy is a protective mechanism in human chondrocytes with mitochondrial dysfunction (3). These observations suggest that autophagy serves an important role to protect chondrocytes from oxidative stress, and additionally supported the theory that pharmacological interventions targeting autophagy may 
prevent cartilage degradation (3). The effect of autophagy on chondrocyte senescence remains to be fully elucidated. Therefore, the current study aimed to culture rat chondrocytes treated with DXM, in order to determine the effect of DXM on chondrocyte senescence and autophagy and the association between these two events.

\section{Materials and methods}

All procedures involving Sprague-Dawley rats were performed under the approval and guidance of the Animal Care and Use Committee at Southern Medical University (Guangzhou, China).

Rat knee chondrocyte culture. A total of 34 3-month-old Sprague-Dawley male rats, ranging in weight between 300 and $340 \mathrm{~g}$, were obtained from the Laboratory Animal Center of Southern Medical University. These rats were anesthetized by $10 \%$ chloral hydrate and sacrificed by cervical dislocation, and the articular cartilage was separated from the femoral condyles and tibial plateaus under a microscope. Cartilage slices were incubated with trypsin $(0.5 \mathrm{mg} / \mathrm{ml})$ (Sigma-Aldrich; Merck Millipore, Darmstadt, Germany) for $30 \mathrm{mins}$ at $37^{\circ} \mathrm{C}$. Subsequent to removal of trypsin, the cartilage slices were incubated with $0.1 \%$ collagenase, type II (Sigma-Aldrich; Merck Millipore) in Dulbecco's modified Eagle's medium (DMEM; Life Technologies; Thermo Fisher Scientific, Inc., Waltham, MA, USA) with 5\% fetal calf serum (FCS; Life Technologies; Thermo Fisher Scientific, Inc.) for $4 \mathrm{~h}$ at $37^{\circ} \mathrm{C}$ with shaking. The isolated chondrocytes were recovered and plated in DMEM supplemented with $10 \% \mathrm{FCS}$ and $1 \%$ penicillin/streptomycin. The chondrocytes were incubated at $37^{\circ} \mathrm{C}$ in a humidified gas mixture containing $5 \% \mathrm{CO}_{2}$ balanced with air. In the current study, all cells used were second-generation chondrocytes. DXM (Sigma-Aldrich; Merck Millipore) was added to the chondrocytes at various concentrations $(0,0.1,1,25$ and $50 \mu \mathrm{g} / \mathrm{ml})$.

3-MA and DXM were added to chondrocytes at various concentrations $(0 \mu \mathrm{g} / \mathrm{ml}, 10 \mathrm{mmol} / \mathrm{l} 3-\mathrm{MA}$, $10 \mathrm{mmol} / \mathrm{l} 3-\mathrm{MA}+25 \mu \mathrm{g} / \mathrm{ml} \mathrm{DXM}$ and $25 \mu \mathrm{g} / \mathrm{ml} \mathrm{DXM})$.

Alcian blue staining. Chondrocytes were fixed with $4 \%$ paraformaldehyde for $30 \mathrm{~min}$ at $37^{\circ} \mathrm{C}$. Chondrocytes were stained with 1\% Alcian Blue 8GX (Sigma-Aldrich; Merck Millipore) dissolved in glacial acetic acid for $30 \mathrm{~min}$ at room temperature. The sections were counterstained with $0.1 \%$ nuclear read dissolved in 5\% aluminium sulfate for $20 \mathrm{sec}$ followed by routine dehydration. The stained sections and cells were washed three times with phosphate-buffered saline (PBS) and observed by light microscopy.

LysoTracker Red staining. Chondrocytes $\left(3 \times 10^{5}\right.$ cells/well) were fixed with various concentrations of DXM for 4 days at $37^{\circ} \mathrm{C}$ in $24-w e l l$ plates and rinsed with DMEM three times. LysoTracker Red culture medium $(66 \mathrm{mM} ; 1 \mathrm{ml})$ was added to each well and then the cells were cultured for $30 \mathrm{~min}$ at $37^{\circ} \mathrm{C}$. Subsequent to washing with PBS three times, the chondrocytes were observed using an inverted fluorescence microscope.

Monodansylcadaverine(MDC)staining.Monodansylcadaverine is a specific in vivo marker for autophagic vacuoles. Chondrocytes
( $3 \times 10^{6}$ cells/well) were fixed at different concentrations of DXM for 4 days at $37^{\circ} \mathrm{C}$ in 6 -well plates and rinsed with PBS three times. Subsequently, chondrocytes were incubated at $37^{\circ} \mathrm{C}$ in $0.05 \mathrm{mM}$ MDC (Sigma-Aldrich; Merck Millipore) for $30 \mathrm{~min}$. Following incubation, chondrocytes were washed three times with $\mathrm{PBS}$ at $37^{\circ} \mathrm{C}$ and fixed for $30 \mathrm{~min}$ in $4 \%$ paraformaldehyde. Following fixation, chondrocytes were washed four times with PBS and observed under a fluorescence microscope. To observe the rate of autophagy, stained chondrocytes were tested using flow cytometry.

Western blotting. Chondrocytes incubated with various concentrations of DXM $(0,0.1,1,25$ and $50 \mu \mathrm{g} / \mathrm{ml})$ were washed with PBS. Total proteins were isolated, and were mixed with a standard protein solution from the Bicinchoninic Acid (BCA) assay kit to produce the BCA working fluid. Each well was filled with the working fluid $(200 \mu \mathrm{l})$ for $15 \mathrm{~min}$ at room temperature. The protein concentration was determined according to the measured optical density values. According to the protein concentration of each group (sample quantity, $30 \mu \mathrm{g}$; total volume, $20 \mu \mathrm{l}$ ), the total amount of protein in each group was calculated. Subsequent to heating with loading buffer (Beyotime Institute of Biotechnology, Haimen, China) and PBS at $100^{\circ} \mathrm{C}$ for $5 \mathrm{~min}$, protein samples were prepared. Equal quantities $(30 \mu \mathrm{g})$ of protein from each sample were separated using $12 \%$ sodium dodecyl sulfate-polyacrylamide gel electrophoresis and were transferred onto polyvinylidene difluoride membranes (Bio-Rad Laboratories, Inc., Hercules, CA, USA). Following blocking with $5 \%$ nonfat milk, the membranes were incubated with the following rabbit anti-rat primary antibodies overnight at $4^{\circ} \mathrm{C}$ : Polyclonal anti-beclin-1 (1:1,000; Abcam, Cambridge, MA, USA; cat. no. ab55878), mouse monoclonal anti-p62 (1:1,000; Abcam; cat. no. ab56416), rabbit polyclonal anti-LC3 (1:1,000; Cell Signaling Technology, Inc., Danvers, MA, USA), mouse polyclonal anti- $\beta$-actin $(1: 1,000$; Beyotime Institute of Biotechnology; cat. no. AA128). Next, the membranes were incubated with horseradish peroxidase-conjugated secondary antibodies (goat anti-rabbit; 1:2,000; cat. no. A0208; Beyotime Institute of Biotechnology) for $2 \mathrm{~h}$ at $37^{\circ} \mathrm{C}$. The bands were detected using ECL plus reagent (Invitrogen; Thermo Fisher Scientific, Inc.) on an enhanced chemiluminescence detection system (PerkinElmer, Inc., Waltham, MA, USA). In addition, the intensity of the bands was quantified using Alpha Ease FC software, version 4.0 (Alpha Innotech Corporation; ProteinSimple, Santa Clara, CA, USA).

$\beta$-galactosidase $(\beta$-gal) staining. Chondrocytes were treated with 3-MA and DXM at various concentrations $(0 \mu \mathrm{g} / \mathrm{ml}$, $10 \mathrm{mmol} / \mathrm{l} 3-\mathrm{MA}, 10 \mathrm{mmol} / \mathrm{l} 3-\mathrm{MA}+25 \mu \mathrm{g} / \mathrm{ml} \mathrm{DXM}$ and $25 \mu \mathrm{g} / \mathrm{ml} \mathrm{DXM}$ ) for 24,48 and $72 \mathrm{~h}$; then, chondrocytes were fixed using a Senescence $\beta$-Galactosidase Staining kit for $15 \mathrm{~min}$ at room temperature. Chondrocytes were stained with $\beta$-gal dye for $12 \mathrm{~h}$ and then washed three times. The stained cells were observed using a phase-contrast microscope to determine the percentage of positive cells out of the total chondrocytes under magnification of $\mathrm{x} 200$.

Red fluorescent protein (RFP)-green fluorescent protein (GFP)-light chain 3 (LC3) assay. Prior to treatment with glucocorticoids, chondrocytes were transfected with monomeric 
(m)RFP-GFP-LC3 when the confluence was 50-70\% using RFP-GFP-LC3 adenoviral vectors (HanBio Technology Co., Ltd., Shanghai, China). The multiplicity of infection was 100. The chondrocytes were incubated with the adenovirus in DMEM with no serum for $2 \mathrm{~h}$ at $37^{\circ} \mathrm{C}$. The transfected chondrocytes were incubated with $10 \%$ DMEM supplemented with fetal bovine serum overnight prior to glucocorticoid treatment to eliminate the effect of starvation on an autophagic level. Following treatment with different doses of glucocorticoids for 4 days, autophagosomes and autolysomes in chondrocytes were observed under a confocal microscope (SP8; Leica Microsystems GmbH, Wetzlar, Germany).

Statistical analysis. Statistical analyses were performed using SPSS statistical software, version 16 (SPSS, Inc., Chicago, IL, USA). One-way analysis of variance was used to analyze the differences between groups. Tukey's significance test was used to detect differences between two groups. $\mathrm{P}<0.05$ was considered to indicate a statistically significant difference.

\section{Results}

Chondrocyte identification. Chondrocytes were stained with toluidine blue. The extracellular matrices of the chondrocytes were stained blue and the nuclei were stained dark blue, which is consistent with typical chondrocyte characteristics (Fig. 1A).

DXM promote chondrocyte autophagy. Chondrocytes were incubated with different concentrations of DXM for 4 days and stained with LysoTracker and MDC to observe autophagic vacuoles. Compared with the control group, only a small number of chondrocytes displayed LysoTracker-positive staining (Fig. 1B). It was observed that the intensity of LysoTracker-positive cells increased markedly with increasing concentrations of DXM (Fig. 1B). The majority of chondrocytes were incubated with $50 \mu \mathrm{g} / \mathrm{ml}$ DXM for 4 days and were positively stained with LysoTracker. Therefore, DXM is able to promote autophagous vesicles in the chondrocytes.

The results of LysoTracker staining were similar to that of MDC staining. In the control group, only certain cells were stained positive for MDC (Fig. 2A) and the intensity of MDC staining in the cells was markedly increased with increasing concentrations of DXM. The MDC-stained chondrocytes were observed by flow cytometry, and it was demonstrated that the cells that were incubated with the different concentrations of DXM had higher incidences of autophagy compared with the control group after 4 days $(\mathrm{P}<0.05)$. In the $50 \mu \mathrm{g} / \mathrm{ml}$ group, autophagy occurred with an average incidence of $56.74 \%$.

RFP-GFP-LC3 assay. Autophagy is a recycling process including the maturation of autophagosomes, fusion of autophagosomes and lysosomes, autolysosome formation and degradation. The total process is termed autophagic flux. In vitro, autophagic flux can be determined by the transfection of an adenovirus harboring mRFP-GFP-LC3. Subsequent to transfection, the autophagosomes in cells are presented as yellow dots (the combination of red and green fluorescence), and the autolysosomes are presented as red dots (the extinction of GFP in the acid environment of lysosomes). Following culture for 4 days, the yellow autophagosomes were increased in the cytoplasm of chondrocytes with increasing DXM dose (Fig. 2G), indicating the stimulatory role of DXM on autophagic flux in chondrocytes.

DXM upregulates autophagy-associated proteins. To determine the role of DXM in regulating chondrocyte autophagy, western blotting was conducted in order to observe the expression levels of LC3, beclin-1 and P62. When chondrocytes were incubated with different concentrations of DXM for 2 days, no significant differences in the expression levels of LC3-II/ $\beta$-actin in the different groups were observed. However, after 4 days, the expression levels of LC3-II/ $\beta$-actin were significantly increased with increasing concentrations of DXM, and after 6 days, the expression levels of LC3-II/ $\beta$-actin were reduced at the highest concentration of DXM (Fig. 3). Changes in the expression levels of P62 and beclin-1 were also similar, however, notably, the expression levels of P62 reduced with increasing concentrations of DXM after 2 days.

DXM inhibits the mechanistic target of rapamycin (mTOR) signaling pathway. The mechanism of activating autophagy is predominantly associated with mTOR-dependent and mTOR-independent pathways, with p70S6K and 4EBP1 involved as downstream effectors in mTOR-dependent pathways. In the 1 and $25 \mu \mathrm{g} / \mathrm{ml}$ treatment groups, the expression levels of P-p70S6K and 4EBP1 were reduced when compared with the control group, and the difference was identified to be statistically significant (Fig. 3G), indicating that DXM inhibits autophagy through an mTOR-dependent pathway.

The role of DXM and autophagy in chondrocyte senescence. When the cells become senescent, $\beta$-gal-positive staining occurs. The proportion of $\beta$-gal-positive chondrocytes significantly increased with time upon treatment with $25 \mu \mathrm{g} / \mathrm{ml}$ glucocorticoid, and was greatest following treatment for $72 \mathrm{~h}$ (Fig. 4A-E). However, the proportion of $\beta$-gal-positive chondrocytes increased significantly when 3-MA, which can inhibit autophagy, was added in comparison with the DXM group alone after $72 \mathrm{~h}$. Furthermore, 3-MA alone was not able to increase the proportion of $\beta$-gal-positive chondrocytes (Fig. 4F).

\section{Discussion}

In the current study, it was investigated whether DXM is able to inhibit chondrocyte autophagy through an mTOR-dependent pathway and induce senescence. Senescence activated by DXM was observed to be inhibited by autophagy, and it was identified that chondrocyte senescence increased with the inhibition of autophagy.

DXM is widely used to relieve a variety of symptoms caused by OA, however long-term and repeated treatment often results in complications $(12,13)$. DXM can lead to chondrocyte apoptosis, growth inhibition, and a reduction in biological activity $(13,14)$. Apoptosis may reduce the number of chondrocytes and reduce the extracellular 
A

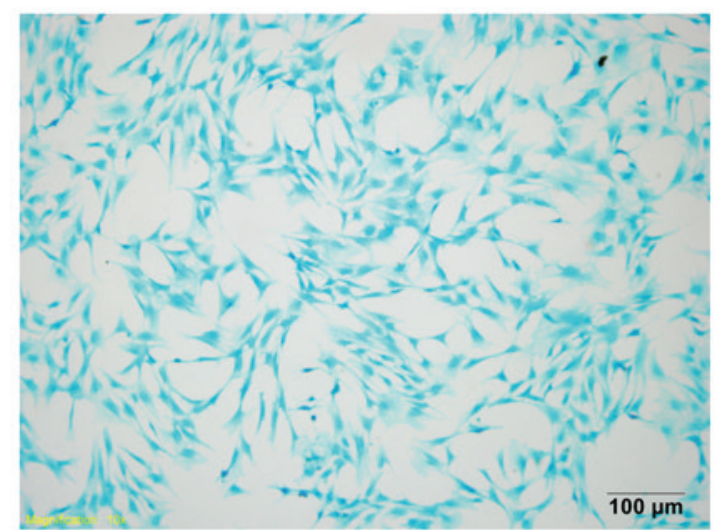

B

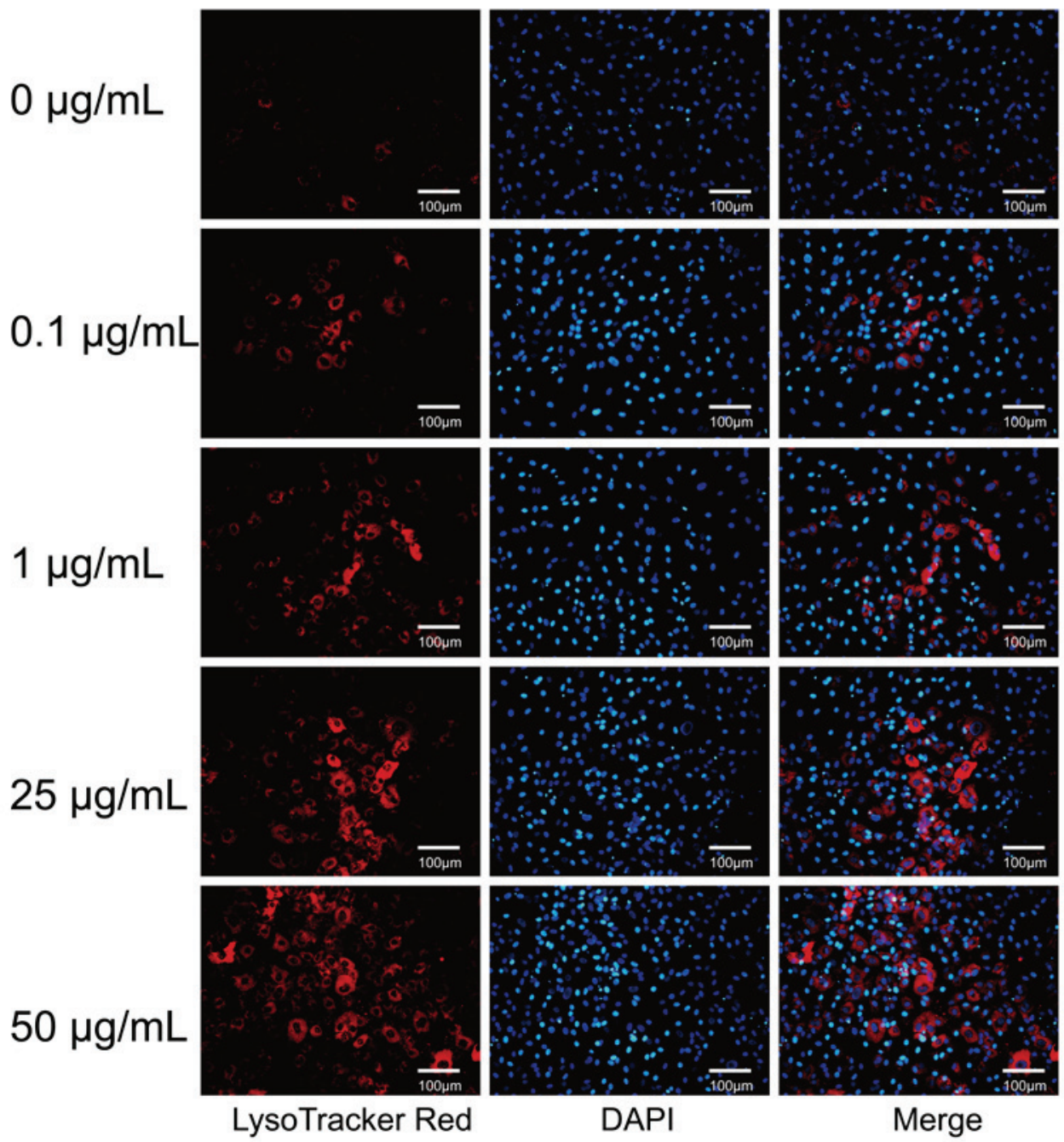

Figure 1. (A) Chondrocytes were stained with alcian blue staining. (B )The LysoTracker Red staining of chondrocytes treated with dexamethasone at different concentrations for 4 days. A dose-dependent increase in the intensity of LysoTracker Red staining in chondrocytes stimulated with dexamethasone was observed. Scale bar, $100 \mu \mathrm{m}$.

matrix, including proteoglycan and collagen type II, thus it is suggested that chondrocyte apoptosis is an important factor in the pathogenesis of OA (15). While autophagy is a programmed cell death program similar to apoptosis, the effects of glucocorticoid treatment on chondrocyte autophagy remain to be investigated.

Autophagy is not only similar to apoptosis but also has a protective effect on chondrocytes in OA $(2,3,16)$. Autophagy has gained research focus due to its potential in regulating the aging process. A previous study has reported that the activity of autophagy was reduced in senescent tissues (17). When cells encounter certain stress situations or bacterial invasion, double membranous vesicles called autophagosomes are formed. These autophagosomes either fuse with endosomes or lysosomes, leading to the formation of autolysosomes. The process begins with the formation of a double membranous 

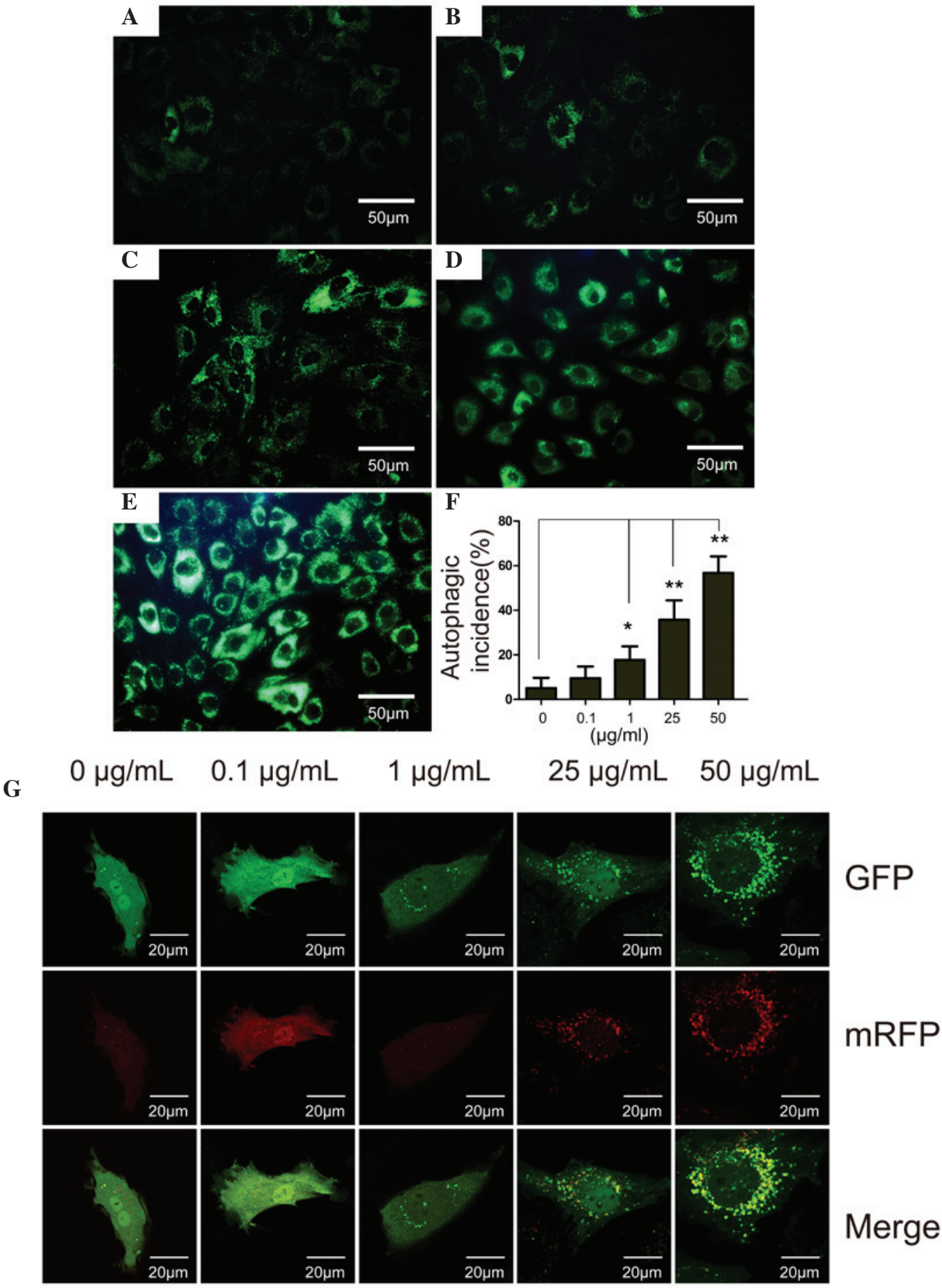

Figure 2. The MDC staining, flow cytometry analysis and RFP-GFP-LC3 assay of chondrocytes treated with DXM. MDC staining of chondrcytes treated with different concentrations of DXM: (A) $0 \mu \mathrm{g} / \mathrm{ml}$, (B) $0.1 \mu \mathrm{g} / \mathrm{ml}$, (C) $1 \mu \mathrm{g} / \mathrm{ml}$, (D) $25 \mu \mathrm{g} / \mathrm{ml}$ and (E) $50 \mu \mathrm{g} / \mathrm{ml}$. Scale bar, $50 \mu \mathrm{m}$. (F) Autophagic incidence analyzed by flow cytometry. Mean \pm standard deviation. $\mathrm{n}=3$. ${ }^{*} \mathrm{P}<0.05$ vs. control; ${ }^{* *} \mathrm{P}<0.01$ vs. control. (G) Prior to DXM treatment, chondrocytes were transfected with RFP-GFP-LC3 adenoviral vectors. A dose-dependent increase in the number of autophagosomes formed was observed in the DXM-treated chondrocytes. MDC, monodansylcadaverine; GFP, green fluorescent protein; RFP, red fluorescent protein; DXM, dexamethasone; LC3, light chain 3.

phagophore that elongates into an autophagosome, during which cellular material becomes enclosed (18). In this way, autophagy can transport larger organelles or pathogens. In vitro, autophagy can inhibit chondrocyte apoptosis and prevent degradation of the extracellular matrix induced by interleukin $1 \beta$ (19). In vivo, it remains unclear whether intraperitoneal injection of rapamycin or local intra-articular injection of rapamycin can promote chondrocyte autophagy and reduce the progress of degeneration of articular cartilage $(2,4)$. In the current study, it was identified that DXM significantly activates chondrocyte autophagy, thus it was hypothesized that autophagy may be compensatory to the chondrocyte damage induced by DXM.

mTOR is one of the components of mTOR complex 1 (mTORC1) and serves as a key switch in regulating autophagy, thus the autophagic signal pathway can be divided into 
A

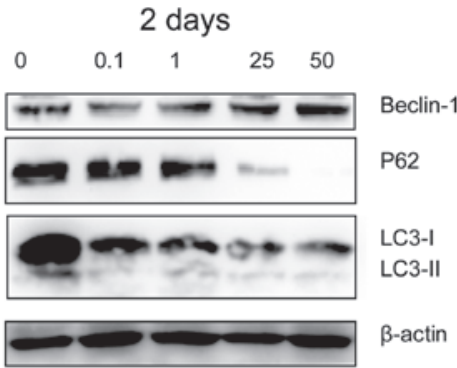

C

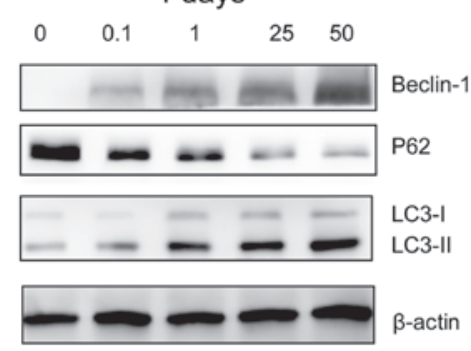

$\mathbf{E}$

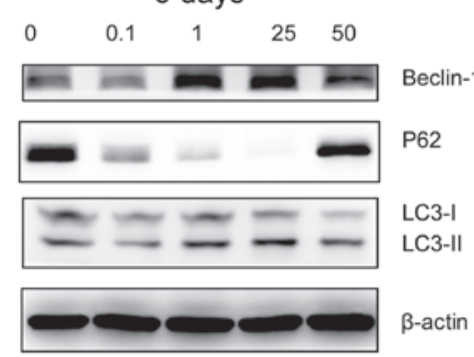

G

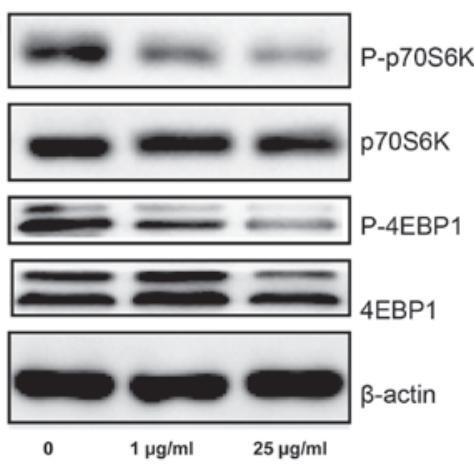

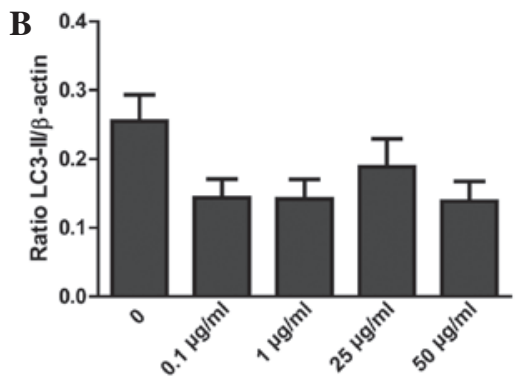

D
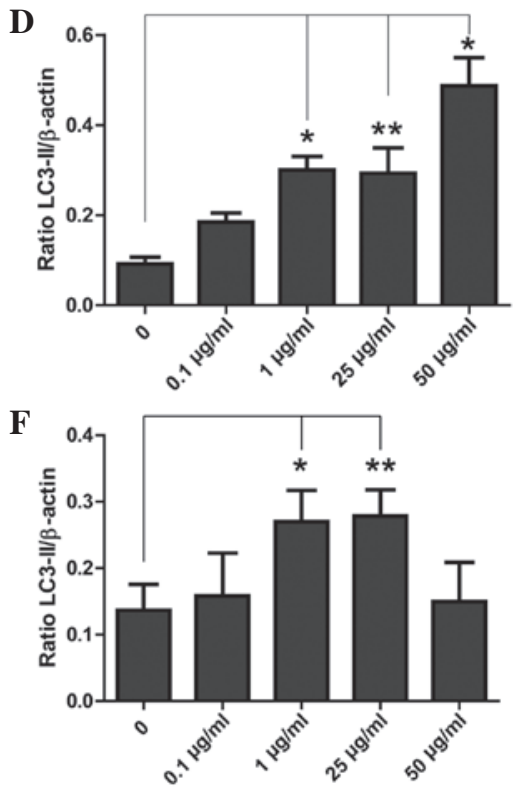

H
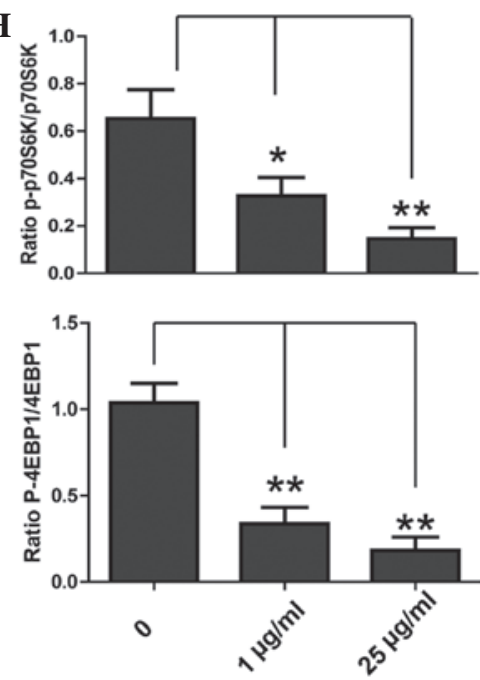

Figure 3. The expression of autophagy-associated protein and mechanistic target of rapamycin pathway-associated proteins in chondrocytes treated with dexamethasone. (A, C and E) The expression of beclin-1, P62 and LC3 in chondrocyte analyzed by western blotting. (B, D and F) The corresponding optical densities of LC3-II/ß-actin were analyzed. (G) The expression of P-p70S6 K and P-4EBP1 in chondrocytes analyzed by western blotting. (H and I) The corresponding optical densities of P-p70S6K/p70S6K and P-4EBP1/4EBP1 were analyzed. Data are presented as the mean \pm standard deviation; $\mathrm{n}=3$; ${ }^{*} \mathrm{P}<0.05$ vs. control; ${ }^{* *} \mathrm{P}<0.01$ vs. control. LC3, light chain 3 ; P-, phosphorylated.

mTOR-dependent and mTOR-independent pathways (20). Commonly, the stimulation of autophagy begins with the inhibition of mTOR, and the most important of two downstream targets of mTORC1 are p70S6K and 4EBP1. It was identified that DXM can reduce p70S6K and 4EBP1 phosphorylation and activity, and thus, DXM activates autophagy in chondrocytes through an mTOR-dependent pathway. Similarly, glucosamine, which is the current treatment for
OA, also activates autophagy through an mTOR-dependent pathway (21). These results demonstrate that the predominant signal pathway of autophagy activation in chondrocytes is mTOR-dependent.

DXM can reduce cell growth and inhibit cell activity. Poulsen et al (22) identified that DXM can reduce tendon cell growth and induce senescence. However, whether DXM can induce chondrocyte senescence remains to be determined. 

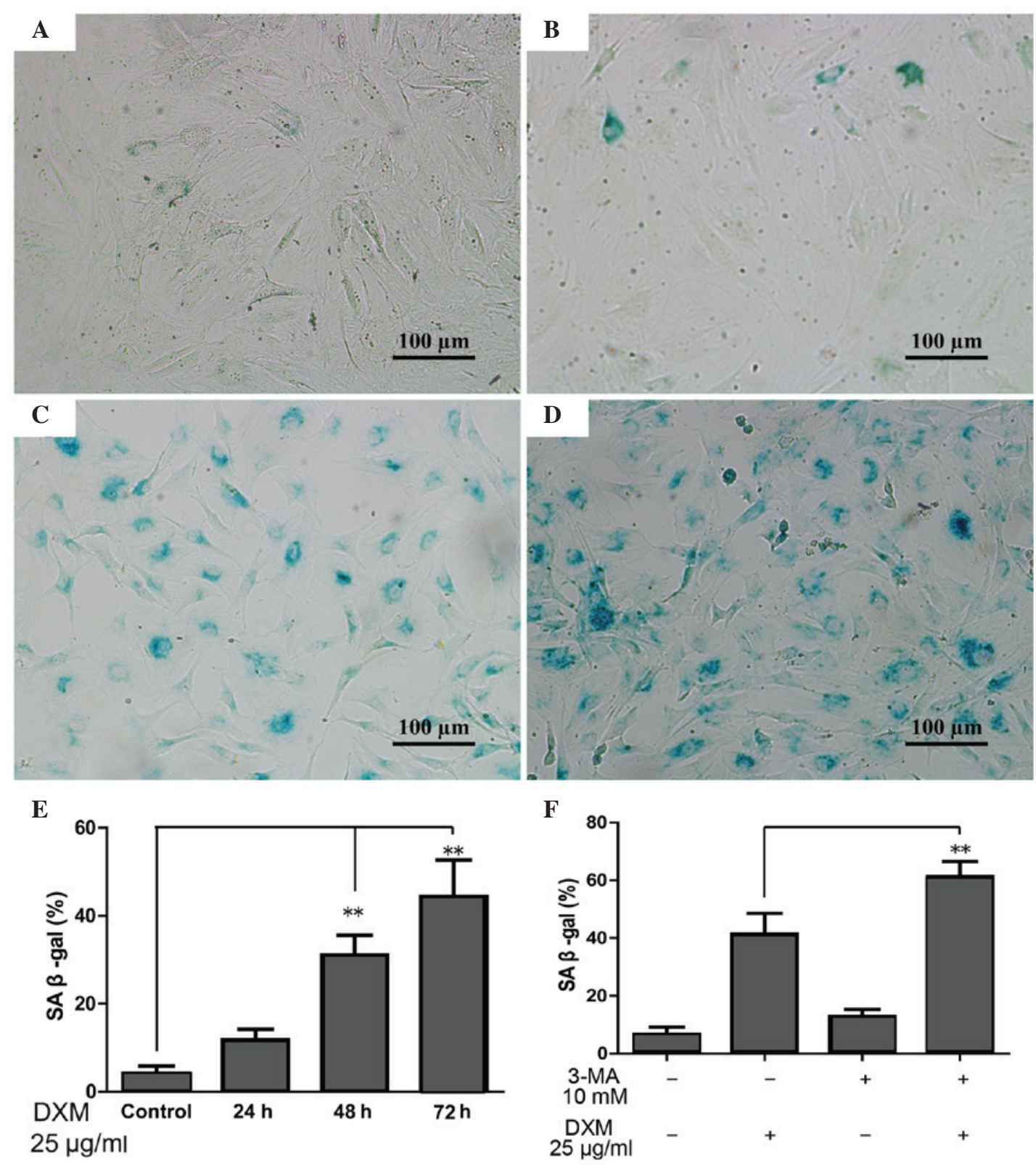

Figure 4. $\beta$-gal staining of the chondrocytes treated with DXM and/or 3-MA. (A-D) $\beta$-gal staining of the chondrocytes treated with $25 \mu \mathrm{g} / \mathrm{ml}$ for 25,48 and $72 \mathrm{~h}$. (E) The quantitative analysis of the incidence of the cells with positive $\beta$-gal staining. ${ }^{* *} \mathrm{P}<0.01$ vs. control. (F) The chondrocytes treated with DXM and/or 3-MA. The incidence of positive $\beta$-gal staining was analyzed. ${ }^{* *} \mathrm{P}<0.01$ vs. cells treated with DXM alone. Data are presented as the mean \pm standard deviation; $\mathrm{n}=3$. $\beta$-galactosidase, $\beta$-gal; DXM, dexamethasone; 3-MA, 3-methyladenine; SA, senescence-associated.

When cell-cycle progression is arrested, the cell can be in a static state or become senescent. When the cell senesces, cell proliferation stops, however its volume loss continues to increase, resulting in aging cells being larger than normal cells (23). In the current study, it was identified that DXM can promote chondrocyte senescence and that this effect is more marked as time progresses. Chondrocyte senescence has been considered to be a correlative factor for OA, thus it is suggested that the long-term use of glucocorticoid-induced senescence may be an explanation for chondrocytes undergoing degeneration and necrosis.

The association between autophagy and senescence remains controversial. Kamalakannan et al (24) identified that autophagy can prevent mononuclear cell senescence caused by heat shock protein, however it induces bronchial epithelial cells to undergo senescence caused by oxidative stress (25). For chondrocytes, senescence was activated when 3-MA was used to inhibit autophagy, which indicates that glucocorticoid-induced autophagy may be a compensatory protective effect against senescence.

In conclusion, the current study identified that DXM can inhibit chondrocyte autophagy through an mTOR-dependent pathway and induce senescence. Autophagy may therefore serve as a protective process against senescence.

\section{Acknowledgements}

The present study was supported by a grant from the WenZhou Science and Technology Research Project (grant no. Y20140582). 


\section{References}

1. Reyes C, Garcia-Gil M,Elorza JM, Mendez-Boo L, Hermosilla E, Javaid MK, Cooper C, Diez-Perez A, Arden NK, Bolibar B, et al Socio-economic status and the risk of developing hand, hip or knee osteoarthritis: A region-wide ecological study. Osteoarthritis Cartilage 23: 1323-1329, 2015.

2. Takayama K, Kawakami Y, Kobayashi M, Greco N, Cummins JH, Matsushita T, Kuroda R, Kurosaka M, Fu FH and Huard J: Local intra-articular injection of rapamycin delays articular cartilage degeneration in a murine model of osteoarthritis. Arthritis Res Ther 16: 482, 2014.

3. López de Figueroa P, Lotz MK, Blanco FJ and Caramés B Autophagy activation and protection from mitochondrial dysfunction in human chondrocytes. Arthritis Rheumatol 67 966-976, 2015.

4. Caramés B, Hasegawa A, Taniguchi N, Miyaki S, Blanco FJ and Lotz M: Autophagy activation by rapamycin reduces severity of experimental osteoarthritis. Ann Rheum Dis 71: 575-581, 2012.

5. Zhang M, Zhang J, Lu L, Qiu ZY, Zhang X, Yu SB, Wu YP and Wang MQ: Enhancement of chondrocyte autophagy is an early response in the degenerative cartilage of the temporomandibular joint to biomechanical dental stimulation. Apoptosis 18: 423-434, 2013.

6. Martinet W, De Meyer GR, Herman AG and Kockx MM: Amino acid deprivation induces both apoptosis and autophagy in murine C2C12 muscle cells. Biotechnol Lett 27: 1157-1163, 2005.

7. Park EY and Park JB: High glucose-induced oxidative stress promotes autophagy through mitochondrial damage in rat notochordal cells. Int Orthop 37: 2507-2514, 2013.

8. Stevens DG, Beharry R, McKee MD, Waddell JP and Schemitsch EH: The long-term functional outcome of operatively treated tibial plateau fractures. J Orthop Trauma 15: 312-320, 2001.

9. Martin JA, Brown TD, Heiner AD and Buckwalter JA: Chondrocyte senescence, joint loading and osteoarthritis. Clin Orthop Relat Res (427 Suppl): S96-S103, 2004

10. Martin JA and Buckwalter JA: Telomere erosion and senescence in human articular cartilage chondrocytes. J Gerontol A Biol Sci Med Sci 56: B172-B179, 2001.

11. Buckwalter JA, Martin J and Mankin HJ: Synovial joint degeneration and the syndrome of osteoarthritis. Instr Course Lect 49: 481-489, 2000.

12. Tu Y, Xue H, Francis W, Davies AP, Pallister I, Kanamarlapudi V and Xia Z: Lactoferrin inhibits dexamethasone-induced chondrocyte impairment from osteoarthritic cartilage through up-regulation of extracellular signal-regulated kinase $1 / 2$ and suppression of FASL, FAS, and Caspase 3. Biochem Biophys Res Commun 441: 249-255, 2013.
13. Zaman F, Chrysis D, Huntjens K, Chagin A, Takigawa M, Fadeel B and Sävendahl L: Dexamethasone differentially regulates Bcl-2 family proteins in human proliferative chondrocytes: Role of pro-apoptotic Bid. Toxicol Lett 224: 196-200, 2014.

14. Hong D, Chen HX, Yu HQ, Wang C, Deng HT, Lian QQ and Ge RS: Quantitative proteomic analysis of dexamethasone-induced effects on osteoblast differentiation, proliferation, and apoptosis in MC3T3-E1 cells using SILAC. Osteoporos Int 22: 2175-2186, 2011.

15. Aigner T, Hemmel M, Neureiter D, Gebhard PM, Zeiler G, Kirchner T and McKenna L: Apoptotic cell death is not a widespread phenomenon in normal aging and osteoarthritis human articular knee cartilage: A study of proliferation, programmed cell death (apoptosis), and viability of chondrocytes in normal and osteoarthritic human knee cartilage. Arthritis Rheum 44: 1304-1312, 2001.

16. Caramés B, Olmer M, Kiosses WB and Lotz MK: The relationship of autophagy defects and cartilage damage during joint aging in a mouse model. Arthritis Rheumatol 67: 1568-1576, 2015 .

17. Wu X, Won H and Rubinsztein DC: Autophagy and mammalian development. Biochem Soc Trans 41: 1489-1494, 2013.

18. Levine B and Kroemer G: Autophagy in the pathogenesis of disease. Cell 132: 27-42, 2008.

19. Sasaki H, Takayama K, Matsushita T, Ishida K, Kubo S, Matsumoto T, Fujita N, Oka S, Kurosaka M and Kuroda R: Autophagy modulates osteoarthritis-related gene expressions in human chondrocytes. Arthritis Rheum 64: 1920-1928, 2012.

20. Alayev A and Holz MK: mTOR signaling for biological control and cancer. J Cell Physiol 228: 1658-1664, 2013.

21. Caramés B, Kiosses WB, Akasaki Y, Brinson DC, Eap W, Koziol J and Lotz MK: Glucosamine activates autophagy in vitro and in vivo. Arthritis Rheum 65: 1843-1852, 2013.

22. Poulsen RC, Watts AC, Murphy RJ, Snelling SJ, Carr AJ and Hulley PA: Glucocorticoids induce senescence in primary human tenocytes by inhibition of sirtuin 1 and activation of the p53/p21 pathway: In vivo and in vitro evidence. Ann Rheum Dis 73: 1405-1413, 2014.

23. Muller M: Cellular senescence: Molecular mechanisms, in vivo, significance and redox considerations. Antioxid Redox Signal 11: 59-98, 2009.

24. Kamalakannan V, Shiny A, Babu S and Narayanan RB: Autophagy protects monocytes from wolbachia heat shock protein 60-induced apoptosis and senescence. PLoS Negl Trop Dis 9: e0003675, 2015.

25. Ito S, Araya J, Kurita Y, Kobayashi K, Takasaka N, Yoshida M, Hara H, Minagawa S, Wakui H, Fujii S, et al: PARK2-mediated mitophagy is involved in regulation of HBEC senescence in COPD pathogenesis. Autophagy 11: 547-559, 2015. 